\title{
Criminologie
}

\section{Prescription médicale de stupéfiants et délinquance}

\section{Résultats des essais suisses}

\section{Marcelo F. Aebi, Denis Ribeaud et Martin Killias}

Volume 32, numéro 2, automne 1999

La justice des mineurs

URI : https://id.erudit.org/iderudit/004707ar

DOI : https://doi.org/10.7202/004707ar

Aller au sommaire du numéro

\section{Éditeur(s)}

Les Presses de l'Université de Montréal

ISSN

0316-0041 (imprimé)

1492-1367 (numérique)

Découvrir la revue

Citer cet article

Aebi, M. F., Ribeaud, D. \& Killias, M. (1999). Prescription médicale de stupéfiants et délinquance : résultats des essais suisses. Criminologie, 32(2),

127-148. https://doi.org/10.7202/004707ar
Résumé de l'article

Suite à l'aggravation du problème de la toxicomanie pendant les années 1980 la Suisse a commencé à appliquer une politique de la drogue diversifiée dont l'un des piliers s'inspire de l'approche de la réduction des risques. L'un des buts de cette politique est notamment la diminution des dommages collatéraux à la toxicomanie, telles certaines maladies infectieuses ou la délinquance liée à l'acquisition de drogues.Dans ce contexte, un programme de prescription médical d'opiacés a été mis sur pied. Ce programme s'adresse à des héroïnomanes fortement dépendants et pour lesquels d'autres formes de traitement n'ont pas eu de succès. S'agissant d'un type de traitement novateur, il fit l'objet d'une stricte évaluation scientifique. Le présent article résume les résultats de cette évaluation pour le domaine de la délinquance. Les analyses se basent autant sur des interviews de délinquance et de victimisation autoreportées, menées à intervalles de 6 mois avec les personnes traitées, que sur les données des registres de police et du casier judiciaire central de ces mêmes personnes. Les résultats indiquent pour tous les types de données une diminution massive des taux de prévalence et d'incidence de la criminalité liée à l'acquisition de drogues. Les taux de victimisation présentent une diminution similaire. Il est démontré que ces deux phénomènes sont étroitement liés.
Ce document est protégé par la loi sur le droit d'auteur. L'utilisation des services d’Érudit (y compris la reproduction) est assujettie à sa politique d'utilisation que vous pouvez consulter en ligne.

https://apropos.erudit.org/fr/usagers/politique-dutilisation/ 


\title{
Prescription médicale de stupéfiants et délinquance
}

\author{
Résultats des essais suisses
}

\author{
Marcelo F. Aebi \\ professeur remplaçant \\ Institut de police scientifique et de criminologie \\ Université de Lausanne - Suisse \\ marcelofernando.aebi@ipsc.unil.ch \\ Denis Ribeaud \\ chercheur \\ Institut de police scientifique et de criminologie \\ Université de Lausanne - Suisse \\ denis.ribeaud@ipsc.unil.ch
}

\author{
Martin Killias \\ professeur \\ Institut de police scientifique et de criminologie \\ Université de Lausanne • Suisse \\ martin.killias@ipsc.unil.ch
}

RÉSUMÉ - Suite à l'aggravation du problème de la toxicomanie pendant les années 1980, la Suisse a commencé à appliquer une politique de la drogue diversifiée dont l'un des piliers s'inspire de l'approche de la réduction des risques. L'un des buts de cette politique est notamment la diminution des dommages collatéraux à la toxicomanie, telles certaines maladies infectieuses ou la délinquance liée à l'acquisition de drogues.Dans ce contexte, un programme de prescription médical d'opiacés a été mis sur pied. Ce programme s'adresse à des héroïnomanes fortement dépendants et pour lesquels d'autres formes de traitement n'ont pas eu de succès. S'agissant d'un type de traitement novateur, il fit l'objet d'une stricte évaluation scientifique.Le présent article résume les résultats de cette évaluation pour le domaine de la délinquance. Les analyses se basent autant sur des interviews de délinquance et de victimisation autoreportées, menées à intervalles de 6 mois avec les personnes traitées, que sur les données des registres de police et du casier judiciaire central de ces mêmes personnes. Les résultats indiquent pour tous les types de données une diminution massive des taux de prévalence et d'incidence de la criminalité liée à l'acquisition de drogues. Les taux de victimisation présentent une diminution similaire. Il est démontré que ces deux phénomènes sont étroitement liés. 
ABSTRACT - Following the worsening of drug-addiction problems during the 1980s, Switzerland started to apply a diversified drug policy based partly on the harm reduction approach. In this context, a program of medical prescription of opiates - including heroin - started, on an experimental basis, in 1994. Eligibility was limited to drug addicts over twenty years of age, who had been using illegal drugs for at least two years, displayed signs of health or social deterioration, and for whom other treatments had failed. An evaluation was performed on the effects of the program on criminal involvement of the subjects treated. In the evaluation, several measures were used to compare offence rates before and during the prescription period, namely : records of convictions, police records and extensive interviews (conducted every six months) using instruments measuring self-reported delinquency and victimisation. The results showed substantial drops in the prevalence and incidence of delinquency. The rates of victimisation presented a similar reduction. It was also shown that delinquency and victimisation are closely related.

\section{Historique des essais avec prescription de stupéfiants en Suisse}

Avec une population d'environ sept millions d'habitants, la Suisse compte, d'après des estimations officielles, environ 30000 toxicomanes dépendants des drogues dures, notamment l'héroïne et la cocaïne (OFSP, 1997). Le nombre de ces toxicomanes a fortement augmenté pendant les années 1980 - les mêmes sources signalent qu'ils étaient 5700 en 1979 — et semble s'être stabilisé dans les années 1990. Même si dans ce domaine il est difficile de procéder à des comparaisons internationales parce que les taux de toxicomanes se fondent sur des estimations établies selon différents critères dans chaque pays, il semble que le taux suisse est légèrement plus élevé que celui de ses pays voisins (BorstEilers et al., 1995 : Reuband,1998 ; Killias et Ribeaud, 1999) ${ }^{1}$.

L'augmentation du nombre de toxicomanes a été accompagnée dans les grandes villes suisses d'une augmentation des délits contre la propriété, notamment des vols à l'arraché et des cambriolages. À ce sujet, il est nécessaire de rappeler que — malgré le fait que les relations

1. En revanche, des comparaisons peuvent être faites dans le domaine de la thérapie. Ainsi, d'après l'Office fédéral de la Santé publique (OFSP, 1997), « la France, forte de 60 millions d'habitants, a le même nombre de places de thérapie qu'en Suisse et cinq fois moins de places de traitement à la méthadone ». Les mêmes sources signalent qu'en 1995 environ 14000 personnes suivaient un traitement à la méthadone en Suisse. 
temporelles entre toxicomanie et délinquance sont multiples et complexes - plusieurs recherches indiquent que, règle générale, la délinquance précède la consommation de drogues dures (Ball et al., 1981 ; Kreuzer et al., 1991 ; Killias et al., 1994a ; Grapendaal et al., 1995) mais il semble établi que l'interaction entre ces deux phénomènes conduit à une augmentation démesurée de la délinquance. En effet, étant donné le coût des drogues dures, il devient à long terme pratiquement impossible pour les toxicomanes de se procurer par des voies légales l'argent nécessaire pour acheter la quantité de drogue dont ils ont besoin. Ainsi, les chercheurs signalent régulièrement que les toxicomanes sont responsables d'un grand nombre de délits, quoique peu de données empiriques soient disponibles sur ce sujet.

En ce qui concerne la ville de Zurich, une recherche basée sur les affaires élucidées indique qu'environ trois vols à l'arraché sur quatre et un tiers des cambriolages commis entre 1992 et 1995 étaient motivés par le besoin de se procurer de l'argent pour acheter des drogues (Killias et Uchtenhagen 1995). Dans cette ville, la situation était particulièrement grave du fait de l'existence d'une scène ouverte de la drogue ${ }^{2}$ qui agissait comme pôle d'attraction pour de nombreux toxicomanes venus d'autres localités. Une première fermeture de cette scène au Platzspitz de Zurich en 1992 n'eut comme conséquence que son déplacement vers la gare désaffectée du Letten. Cette deuxième scène fut fermée en 1994, mais cette fois-ci sa fermeture fut accompagnée de plusieurs mesures, parmi lesquelles la possibilité pour les toxicomanes de participer à un programme de prescription médicale d'héroïne. Ce programme était le fruit d'une longue évolution de la politique suisse en matière de drogues qui s'est soldée, dans les années 1990, par l'adoption de la politique dite des quatre piliers ${ }^{3}$.

L'idée à la base de cette politique est d'essayer de gérer le phénomène de la toxicomanie en utilisant plusieurs stratégies complémentaires. Ces stratégies (« piliers ») sont les suivantes :

- prévention ;

- thérapie ;

- réduction des risques et aide à la survie ;

- répression.

2. Les scènes ouvertes (lieux de rencontre des toxicomanes) avaient tout d'abord été tolérées en croyant qu'il était mieux de regrouper les toxicomanes afin de maintenir un certain contrôle et de pouvoir offrir une assistance.

3. Pour un historique de cette évolution, voir Boggio et al. (1997). 
La prescription médicale de stupéfiants s'inscrit dans le pilier « réduction des risques» tout en faisant aussi partie du pilier «thérapie ». Elle fait suite au constat de l'échec des politiques axées uniquement sur la répression. Son but est d'assister les toxicomanes qui traversent la phase de dépendance - qui selon des études médicales dure en moyenne 10 ans (OFSP, 1997) — pour essayer de préserver leur état de santé et d'améliorer leur intégration sociale, ce qui devrait augmenter leurs chances de sortir de la drogue par la suite ${ }^{4}$.

En 1992, une ordonnance du Conseil fédéral a donné la base légale pour le programme de prescription médicale de stupéfiants. Cette ordonnance est conforme à la loi suisse sur les stupéfiants et aux conventions internationales qui permettent des essais à caractère scientifique avec ces substances. N'étant pas un pays producteur d'héroïne, la Suisse a dû aussi obtenir une autorisation spéciale de l'Organe international de contrôle des stupéfiants des Nations unies pour pouvoir s'en procurer légalement.

Les conditions d'admission aux essais étaient les suivantes (RihsMiddel, 1994 ; Uchtenhagen, 1997) :

- avoir au moins 20 ans ;

- être dépendant de l'héroïne depuis au moins deux ans ;

- avoir suivi au moins deux autres traitements sans succès ;

- présenter, du fait de la consommation de drogues, des dommages à la santé et/ou sur le plan social ${ }^{5}$.

En tout, le Conseil fédéral a autorisé 1000 places de thérapie - 800 pour l'héroïne, 100 pour la morphine et 100 pour la méthadone par voie intraveineuse - et 18 centres de traitement. Il s'agit de petites structures - aucune ne dépasse 150 patients traités - ce qui assure un suivi de ces derniers et un encadrement médical et psychologique. Les patients doivent consommer la drogue sur place et sous contrôle des autorités du centre de traitement.

4. La première mesure inspirée par la réduction des risques fut l'échange de seringues. Appliquée en Suisse dès le milieu des années 1980, cette mesure permit une réduction radicale du nombre de nouvelles infections au virus VIH et d'hépatites parmi les toxicomanes (Grob, 1993).

5. Ces conditions permettent de constater qu'il s'agit d'un programme destiné à un groupe de toxicomanes très marginal. Les diverses études réalisées confirment que la situation personnelle et sociale des participants était critique. Il est dès lors évident que les essais avec prescription d'héroïne sont loin d'être comparables à la tolérance, voire à la légalisation des substances prescrites. 
Afin d'évaluer l'expérience, un réseau de chercheurs a été mis sur pied et trois domaines de recherche ont été distingués ${ }^{6}$. Le premier porte sur les substances prescrites : il s'agit de questions pharmacologiques et toxicologiques concernant les formes d'application, le dosage et les effets secondaires. Le second domaine couvre l'évaluation des divers centres de traitements par rapport à la sécurité et aux coûts-bénéfices. Le troisième et principal domaine se concentre sur l'évolution des patients par rapport à leur état de santé, leur comportement de dépendance, leur intégration sociale - dont en particulier l'intégration dans le marché du travail et du logement — et par rapport à leurs comportements délinquants. L'Institut de police scientifique et de criminologie de l'Université de Lausanne (IPSC) a été mandaté pour évaluer ce dernier aspect.

\section{Méthodologie de la recherche ${ }^{7}$}

Afin de mesurer l'effet de la prescription médicale de stupéfiants sur la délinquance des toxicomanes traités, il a fallu déterminer le degré d'implication de ces derniers dans la criminalité avant et après leur admission au programme. À cet effet, nous avons utilisé des données provenant de sources officielles et d'enquêtes. Les sources officielles utilisées sont les dossiers de police et les extraits du casier judiciaire des participants aux essais. Les données d'enquêtes proviennent de sondages de délinquance autoreportée et de victimisation réalisés avec ces derniers au moment de leur entrée dans le programme et, ensuite, à des intervalles de six mois ${ }^{8}$.

L'avantage d'utiliser une telle palette de mesures réside dans le fait qu'elle permet de saisir la délinquance sous plusieurs angles et de suppléer ainsi aux faiblesses de telle ou telle mesure avec les avantages d'une autre. En outre, les données officielles sont particulièrement importantes pour le suivi des personnes qui ont quitté le programme et ont dès lors cessé de participer aux sondages.

Pour les comparaisons des scores avant et après l'entrée dans le programme, nous avons utilisé le test de Student pour observations

6. Pour une synthèse des résultats de cette évaluation, voir Uchtenhagen (1997).

7. Certaines légères divergences au niveau des chiffres présentés par rapport à d'autres publications sur le même sujet sont dues à la mise à jour des bases de données correspondantes.

8. L'accord d'accès aux données de police et aux extraits de casier judiciaire ainsi que la participation aux divers interviews faisaient partie des conditions d'admission aux essais. 
pairées. Finalement, signalons aussi que lors de la présentation des résultats finals des essais, les personnes participant à ces derniers ont été divisées en deux cohortes selon qu'elles étaient entrées en traitement avant ou après le $1^{\mathrm{er}}$ avril 1995, date à laquelle les essais ont été élargis. L'IPSC a été associé à cette date à la recherche. Cela n'a pas eu d'effet sur les données collectées dans les sources officielles : par contre, les données de sondages ne concernent que les participants entrés en traitement à partir de ce moment (650 personnes; nous disposons de la série complète de trois interviews pour 305 d'entre elles). En outre, les sondages ne portent que sur des personnes traitées à l'héroïne, alors que pour les autres mesures nous disposons aussi des données concernant les personnes traitées à la morphine et à la méthadone intraveineuse. Par la suite, nous nous concentrerons toutefois sur les personnes traitées à l'héroïne9.

\section{La délinquance autoreportée}

Le sondage utilisé lors de cette recherche s'inspire du sondage international de délinquance autoreportée (Junger-Tas et al., 1994). À cette occasion, l'IPSC avait adapté aux caractéristiques helvétiques le questionnaire utilisé (Killias et al., 1994b). Pour la recherche actuelle, le nombre de délits a été réduit de 33 à 12. Il s'agit, d'une part, de délits liés à l'acquisition de drogues (vols, vente de drogues) et, d'autre part, de délits violents.

Le Tableau 1 représente les taux de prévalence, tels qu'ils ont évolué à partir de l'interview $\mathrm{A}$, qui faisait fonction de mesure zéro lors de l'entrée dans le programme, en passant ensuite par la première interview de suivi B-1 (six mois après l'entrée) pour arriver finalement à la seconde interview de suivi B-2 (douze mois après l'entrée). Nous pouvons donc voir le pourcentage de personnes interviewées qui déclarent avoir commis au moins une fois le délit considéré au cours des six mois précédant l'interview respective.

La part de ceux qui avouent avoir commis l'un ou l'autre des délits considérés a fortement diminué après le début du traitement. Lors du deuxième entretien de suivi (B-2), la diminution s'est avérée significa-

9. Le lecteur intéressé trouvera dans Killias et Rabasa (1997) des informations sur les personnes sorties précocement du programme (« drop-outs ») ainsi que sur celles traitées avec d'autres substances. 
T A B LEA U 1

Taux de prévalence de la délinquance autoreportée (en \%) au cours des six mois précédant l'interview respective (12 délits, $\mathrm{N}=305$ )

\begin{tabular}{|l|r|r|r|r|c|}
\hline Type de délit & A & B-1 & B-2 & $\begin{array}{c}\text { Diminution } \\
\text { relative } \\
\text { (A-B2) }\end{array}$ & p (A-B2)* \\
\hline Vol dans un automate & 2,0 & 0,3 & 0,0 & $\left(-100 \% \%^{* *}\right)$ &, $014^{* *}$ \\
Vol à l'étalage & 35,0 & 23,0 & 16,1 & $-54 \%$ & $<, 001$ \\
Pickpocket & 3,6 & 0,0 & 0,0 & $-100 \%$ &, 001 \\
Vol à l'arraché & 1,0 & 0,3 & 0,0 & $\left(-100 \%{ }^{* *}\right)$ & $\mathrm{ns}^{* *}$ \\
Cambriolage & 6,9 & 1,9 & 0,0 & $-100 \%$ & $<, 001$ \\
Recel & 13,1 & 5,6 & 3,9 & $-70 \%$ & $<, 001$ \\
Brigandage & 1,0 & 0,3 & 0,7 & $(-30 \% * *)$ & $\mathrm{ns}^{* *}$ \\
Violence physique & 0,7 & 2,3 & 0,7 & $(-0 \% * *)$ & $\mathrm{ns}^{* *}$ \\
Blessure avec arme & 0,3 & 0,3 & 0,3 & $\left(-0 \% \%^{* *}\right)$ & $\mathrm{ns}^{* *}$ \\
Vente de haschisch & 26,3 & 16,4 & 12,5 & $-52 \%$ & $<, 001$ \\
Vente de drogues dures & 46,9 & 12,1 & 8,2 & $-83 \%$ & $<, 001$ \\
Arnaque lors de la & 6,6 & 2,3 & 1,0 & $-85 \%$ & $<, 001$ \\
\hline
\end{tabular}

* Test de Student (T-test) pour observations pairées.

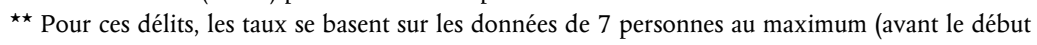
du traitement).

tive pour sept des douze délits en comparaison avec la période avant l'entrée en traitement. Il s'agit des vols à l'étalage, des actes de pickpocket, des cambriolages, des recels, des ventes de différentes drogues ainsi que de l'arnaque lors de la vente de drogues. Les autres délits, avec des taux de prévalence situés au-dessous de $2 \%$, étaient déjà extrêmement rares lors de l'interview de base $(\mathrm{A})$ : dès lors, une évolution significative n'était d'emblée pas envisageable.

Le tableau montre aussi qu'après une première diminution des comportements délinquants dans le courant des six premiers mois du traitement, la tendance s'accentue encore avec le temps. En effet, les taux de neuf des douze délits considérés ont encore diminué entre la première et la seconde interview de suivi.

De plus, on constate que ce sont surtout les délits à but lucratif qui ont diminué. Cela s'explique par la diminution de la pression pour se procurer de l'argent nécessaire à l'aquisition de tupéfiants. Cette dimi- 
nution est de toute évidence attribuable à la prescription de stupéfiants dans le cadre des essais. Ainsi, la part de personnes ayant avoué des actes de pickpocket et de cambriolage a diminué de $100 \%$. La part de personnes vendant des drogues dures et de celles commettant des arnaques lors de telles affaires a aussi considérablement diminué, soit de plus de $80 \%$. La part de receleurs, de vendeurs de cannabis ainsi que de voleurs à l'étalage a diminué de manière moins marquée, quoique toujours sensible, soit de 50 à $70 \%$ environ. En revanche, la part d'auteurs de délits violents, telles lésions corporelles et blessures avec arme — d'ailleurs très rares dans cette population —, n'a pas diminué.

Les participants ont aussi été interrogés sur la fréquence de commission de chaque délit avoué. Cela permet un calcul des taux d'incidence tels qu'ils sont recueillis dans le Tableau 2. Ces taux sont particulièrement importants pour l'estimation du volume de la criminalité dans un espace géographique et temporel donné.

\section{T A B LEA U 2}

Taux d'incidence de la délinquance autoreportée au cours des six mois précédant l'interview respective (12 délits, $\mathrm{N}=305$ )

\begin{tabular}{|c|c|c|c|c|c|}
\hline Type de délit & A & B-1 & B-2 & $\begin{array}{l}\text { Diminution } \\
\text { relative } \\
\text { (A-B2) }\end{array}$ & $p(A-B 2) *$ \\
\hline Vol dans un automate & 0,639 & 0,003 & 0,000 & $(-100 \% * *)$ & $\mathrm{ns}^{* *}$ \\
\hline Vol à l'étalage & 5,639 & 1,705 & 0,845 & $-85 \%$ & $<, 001$ \\
\hline Pickpocket & 0,102 & 0,000 & 0,000 & $-100 \%$ & ,007 \\
\hline Vol à l'arraché & 0,033 & 0,007 & 0,000 & $(-100 \% * *)$ & $\mathrm{ns}^{* *}$ \\
\hline Cambriolage & 0,230 & 0,052 & 0,000 & $-100 \%$ &, 006 \\
\hline Recel & 0,931 & 0,171 & 0,109 & $-88 \%$ &, 001 \\
\hline Brigandage & 0,023 & 0,003 & 0,007 & $\left(-70 \% \%^{\star *}\right)$ & $\mathrm{ns}^{* *}$ \\
\hline Violence physique & 0,013 & 0,020 & 0,010 & $(-23 \% * *)$ & $\mathrm{ns}^{* *}$ \\
\hline Blessure avec arme & 0,003 & 0,000 & 0,007 & $(+133 \% * *)$ & $\mathrm{ns}^{* *}$ \\
\hline Vente de haschisch & 8,960 & 2,129 & 2,162 & $-76 \%$ &, 001 \\
\hline Vente de drogues dures & 25,297 & 3,208 & 2,030 & $-92 \%$ & $<, 001$ \\
\hline $\begin{array}{l}\text { Arnaque lors de la } \\
\text { vente de drogues }\end{array}$ & 0,648 & 0,046 & 0,013 & $-98 \%$ & 014, \\
\hline
\end{tabular}

^ Test de Student (T-test) pour observations pairées.

${ }^{\star \star}$ Pour ces délits, les taux se basent sur les données de 7 personnes au maximum (avant le début du traitement). 
L'évolution des taux d'incidence montre la même tendance que celle des taux de prévalence. Ici aussi, la diminution de la délinquance se poursuit après les six premiers mois de traitement. Notons cependant que l'évolution est encore plus accentuée, avec une diminution statistiquement significative d'environ $90 \%$ entre A et B-2, pour tous les délits liés à l'acquisition de drogues à l'exception des vols à l'arraché, brigandages et vols dans un automate trop rares d'emblée. De nouveau, la diminution n'est pas significative pour les délits peu liés au style de vie des toxicomanes puisque ces actes sont aussi basés sur les données de sept personnes au maximum.

La tendance régressive encore plus marquée pour les taux d'incidence indique que le nombre des délits commis a diminué encore plus fortement que le nombre de personnes délinquantes. Cela implique que les toxicomanes encore délinquants ont commis moins de délits après leur entrée en traitement.

\section{Les victimisations}

Les toxicomanes étant, comme nous venons de le constater, une population particulièrement impliquée dans la délinquance et vivant dans un milieu à risques, il est dès lors probable qu' ils soient souvent victimes de délits. Si le traitement a comme effet un retrait de ce milieu, soit de la scène de la drogue, cela devrait aussi avoir des répercussions en ce qui concerne les victimisations. Le tableau 3 représente les taux de victimisation pour les six délits inclus dans le sondage respectif.

Comme nous pouvons le voir, les expériences de victimisation ont diminué parallèlement à la délinquance autoreportée, même si cela ne s'est pas effectué à tous les niveaux. La diminution est particulièrement marquée pour le brigandage, le vol d'argent et d'effets personnels ainsi que l'arnaque lors de l'achat de drogues, soit des délits étroitement liés au style de vie des toxicomanes et aux risques qu'il comporte. Par contre, pour la violence corporelle et sexuelle ainsi que pour les vols de cycles (véhicules à deux roues), la diminution n'est pas significative. Il s'agit ici de délits qui ne sont pas spécifiques à cette population, comme l'indique aussi le fait qu'ils n'ont été subis que par peu de personnes. Ainsi, il n'est pas étonnant que nous n'y constations pas de diminution significative des taux de victimisation. Au contraire, le fait que la diminution soit différenciée selon les délits souligne la validité de l'instrument utilisé. 
T A B LEA U 3

Taux de prévalence d'expériences de victimisation au cours des six mois précédant l'interview respective ( 6 délits, $\mathrm{N}=305$ )

\begin{tabular}{|l|r|r|r|c|c|}
\hline Type de délit & A & B-1 & B-2 & $\begin{array}{c}\text { Diminution } \\
\text { relative } \\
\text { (A-B2) }\end{array}$ & p (A-B2)* \\
\hline Brigandage & 11,5 & 5,9 & 4,7 & $-59 \%$ &, 001 \\
Violence corporelle & 3,6 & 0,7 & 2,7 & $(-25 \% * *)$ & $\mathrm{ns}^{* *}$ \\
Violence sexuelle & 1,7 & 1,0 & 1,4 & $(-18 \% * *)$ & $\mathrm{ns}^{* *}$ \\
Arnaque - drogue & 55,3 & 18,1 & 16,0 & $-71 \%$ & $<, 001$ \\
Vol (argent, effets) & 23,0 & 11,8 & 13,0 & $-43 \%$ & $<, 001$ \\
Vol de cycles & 14,1 & 9,9 & 9,7 & $-31 \%$ &, 096 \\
\hline
\end{tabular}

* Test de Student (T-test) pour observations pairées.

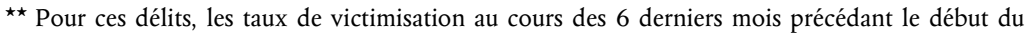
traitement se fondent sur les données de 11 et 5 personnes seulement.

T A B LE A U 4

Taux d'incidence d'expériences de victimisation au cours des six mois précédant l'interview respective ( 6 délits, $\mathrm{N}=305$ )

\begin{tabular}{|l|c|c|c|c|c|}
\hline Type de délit & A & B-1 & B-2 & $\begin{array}{c}\text { Diminution } \\
\text { relative } \\
(\text { A-B2) }\end{array}$ & p (A-B2)* \\
\hline Brigandage & 0,273 & 0,086 & 0,084 & $-69 \%$ &, 004 \\
Violence corporelle & 0,036 & 0,007 & 0,043 & $(+19 \% * *)$ & $n s^{* *}$ \\
Violence sexuelle & 0,092 & 0,013 & 0,013 & $(-86 \% * *)$ & $n \mathrm{~ns}^{* *}$ \\
Arnaque - drogue & 4,465 & 0,492 & 0,572 & $-87 \%$ & $<, 001$ \\
Vol (argent, effets) & 0,792 & 0,234 & $0,180^{* * *}$ & $-77,3 \%$ & $<, 001$ \\
Vol de cycles & 0,201 & 0,125 & 0,128 & $-36 \%$ &, 063 \\
\hline
\end{tabular}

^ Test de Student (T-test) pour observations pairées.

$\star \star$ Pour ces délits, les taux de victimisation au cours des 6 derniers mois précédant le début du traitement (entretien A) se fondent sur les données de 11 et 5 personnes seulement.

${ }^{\star \star \star}$ Une des personnes interviewée prétend avoir été 150 fois victime d'un vol dans cette période. Cette indication semblant peu fiable, ce cas a été éliminé de l'analyse.

Comme pour les délits commis, la fréquence ou incidence des expériences de victimisation est très importante autant pour les victimes elles-mêmes que pour la détermination du volume de criminalité dans l'espace et dans le temps. Pour cette raison, les personnes interviewées ont été priées d'indiquer aussi la fréquence de tels événements. Le Tableau 4 représente les taux correspondants. 
L'évolution de la fréquence de victimisation se présente de manière aussi différenciée que celle des taux de prévalence. Le brigandage, l'arnaque lors de l'achat de drogues et le vol d'argent et d'effets ont diminué sensiblement et de manière significative. Par contre, les rares expériences de violence physique ou sexuelle ainsi que les vols de véhicules à deux roues n'ont pas diminué de manière significative.

\section{Délinquance et victimisation}

Les résultats présentés dans le paragraphe précédent mettent en évidence le fait que les toxicomanes sont très souvent victimes d'infractions. En fait, le taux de victimisation est bien plus élevé dans la population des toxicomanes que dans la population générale (voir Killias et al., à paraître). Comme nous l'avons vu, cela est une conséquence du style de vie toxicomane : dans ce contexte il est intéressant de tester si ce risque est influencé par le degré d'implication dans la délinquance.

En effet, de nombreuses études ont signalé l'existence d'un lien entre la délinquance et le risque de subir soi-même des infractions (Villmow et Stephan, 1983 : 494s. ; Mayhew et Elliott, 1990 ; Killias, 1991 : 249 ; Baron 1997), lien qui découle de toute évidence du mode de vie des délinquants qui se trouvent souvent en contact avec d'autres délinquants (Gottfredson 1984 : 15). Dans le cadre de cette recherche, cela impliquerait que les toxicomanes délinquants seraient plus souvent victimes d'infractions que les toxicomanes non-délinquants.

Ces deux constats — la diminution générale des expériences de victimisation ainsi que la plus forte diminution pour les non-délinquants indiquent un retrait des personnes traitées du milieu toxicomane.

Pour tester cette hypothèse, nous avons comparé les taux de prévalence des victimisations des personnes n'ayant pas avoué de délits (" non-délinquants ») avec ceux des personnes ayant avoué au moins un délit (« délinquants»), et cela, pour les trois périodes d'observation $(\text { Tableau } 5)^{10}$. Cette comparaison est d'autant plus intéressante que la gamme de délits considérée ici recouvre des infractions étroitement liées au style de vie des toxicomanes ainsi que d'autres peu liées à ce dernier.

10. Pour cette comparaison nous utilisons le test d'indépendance du chi-carré qui vise à déterminer si deux variables qualitatives catégorielles (délinquants/non-délinquants et victimes/non-victimes pour chaque délit) observées sur un échantillon sont indépendantes ou non. 
T A B LE A U 5

Prévalence des victimisations sur des périodes de 6 mois avant le début du traitement et après 6 et 12 mois de traitement $(\mathrm{N}=305)$, d'après l'implication dans la délinquance (non-délinquants vs délinquants)

\begin{tabular}{|c|c|c|c|c|c|c|c|c|c|}
\hline \multirow[b]{2}{*}{ Délit subi } & \multicolumn{3}{|c|}{$\begin{array}{l}\text { Avant le début du } \\
\text { traitement }\end{array}$} & \multicolumn{3}{|c|}{$\begin{array}{l}\text { Après } 6 \text { mois de } \\
\text { traitement }\end{array}$} & \multicolumn{3}{|c|}{$\begin{array}{l}\text { Après } 12 \text { mois de } \\
\text { traitement }\end{array}$} \\
\hline & $\begin{array}{l}\text { Non- } \\
\text { délinq. } \\
(N=97)\end{array}$ & $\begin{array}{l}\text { Délinq. } \\
(N=204)\end{array}$ & $p$ & $\begin{array}{c}\text { Non- } \\
\text { délinq. } \\
(N=192)\end{array}$ & $\begin{array}{l}\text { Déli } \\
(N=1\end{array}$ & $p$ & $\begin{array}{c}\text { Non- } \\
\text { délinq. } \\
(N=209)\end{array}$ & $\begin{array}{l}\text { Déli } \\
(N=\end{array}$ & $p$ \\
\hline & $56,7 \%$ & $75,5 \%$ & ** & $28,1 \%$ & $53,6 \%$ & ** & $28,2 \%$ & $54,9 \%$ & ** \\
\hline Brigandage & $6,2 \%$ & $14,1 \%$ & * & $3,6 \%$ & $9,8 \%$ & * & $2,8 \%$ & $9,5 \%$ & * \\
\hline $\begin{array}{l}\text { Violenc } \\
\text { physiq }\end{array}$ & $3,1 \%$ & $3,9 \%$ & ns & $0,5 \%$ & $0,9 \%$ & ns & $1,9 \%$ & $4,8 \%$ & ns \\
\hline $\begin{array}{l}\text { Violence } \\
\text { sexuelle }\end{array}$ & $0,0 \%$ & $2,5 \%$ & ns & $1,0 \%$ & $0,9 \%$ & ns & $0,0 \%$ & $4,9 \%$ & ** \\
\hline $\begin{array}{l}\text { Vol ou arnaque } \\
\text { lors de l'achat } \\
\text { de drogues }\end{array}$ & $46,4 \%$ & $59,5 \%$ & * & $12,0 \%$ & $28,0 \%$ & ** & $11,1 \%$ & $28,6 \%$ & ** \\
\hline Vol de cycles & $11,3 \%$ & $15,6 \%$ & ns & $8,3 \%$ & $12,5 \%$ & ns & $8,9 \%$ & $11,9 \%$ & ns \\
\hline Autres vols & $12,4 \%$ & $28,3 \%$ & $* *$ & $8,9 \%$ & $17,0 \%$ & * & $7,9 \%$ & $26,2 \%$ & ** \\
\hline
\end{tabular}

${ }^{\star} \mathrm{p}<, 05:{ }^{\star \star} \mathrm{p}<, 01$ (tests du chi-carré)

Comme nous pouvons le voir, les taux de prévalence des victimisations parmi les participants délinquants sont en général nettement plus élevés que parmi les non-délinquants. Cette différence semble même s'accroître après le début des essais. Cela confirme donc l'hypothèse du lien étroit entre victimisation et délinquance. Une analyse plus détaillée souligne encore une fois la validité de l'instrument utilisé. En effet, alors que les victimisations étroitement liées au style de vie des toxicomanes - dont en particulier les vols et arnaques lors de l'achat de drogues - présentent des divergences très marquées entre délinquants et non-délinquants, cela n'est pas le cas pour les délits moins liés à ce style de vie, tels les vols de véhicules à deux roues.

Finalement, il nous paraît également important de signaler que l'influence bénéfique du programme se fait sentir sur les deux groupes, puisque chacun d'eux enregistre une diminution des victimisations à partir de l'entrée en traitement. Ces deux constats - la diminution générale des victimisations ainsi que la plus forte diminution pour les non-délinquants - indiquent un retrait progressif mais différentiel des personnes traitées du milieu toxicomane. 


\section{Les contacts avec la police}

Tous les corps de police suisses possèdent des dossiers individuels sur les personnes soupçonnées d'avoir commis un délit ${ }^{11}$. À ce niveau, le système fédéral suisse se reflète dans l'organisation des registres de la police. En effet, il n'existe pas ici un registre central, mais de nombreux registres propres à chaque corps de police. En outre, dans le cas de certaines grandes villes, comme Zurich ou Berne, ces registres sont tenus par la police communale et non cantonale. Ainsi, des informations concernant une même personne peuvent être présentes dans plusieurs registres différents. Pour cette raison, au moment de la récolte des données, tous les registres des corps de police des cantons et des villes dans lesquels un programme de prescription de stupéfiants était en cours ont été consultés ${ }^{12}$. Notons aussi que les informations pertinentes ont été codifiées sur des fiches identifiées par un numéro, de manière à assurer l'anonymat des patients.

Contrairement aux enquêtes, nous disposons à ce stade de données pour les personnes entrées en traitement avant le $1^{\text {er }}$ avril 1995. C'est pour cette raison d'ailleurs que l'échantillon étudié est plus grand que celui qui avait été utilisé dans le cas des données autoreportées présentées auparavant. En revanche, nous ne disposons pas des données du corps de police de Bâle-Ville puisque, dans ce cas-là, le système de classification ne permettait pas de récolter les données dans un laps de temps raisonnable. Par conséquent, les personnes participant à un programme dans cette ville ont été exclues des analyses présentées ci-dessous ${ }^{13}$.

Dans le Tableau 6, on compare pour 604 personnes le nombre de contacts avec la police durant la période de référence de six mois avant

11. Étant donné que la culpabilité ne peut être établie que par un tribunal, nous utiliserons ici plutôt le terme de contact avec la police au lieu de délit pour nous référer aux infractions enregistrées dans ces dossiers.

12. Cela signifie que nous ne disposons probablement pas de tous les dossiers de police des personnes traitées. Deux arguments nous semblent cependant justifier cette démarche. D'une part, le travail engendré par une saisie exhaustive, soit auprès de 28 corps de police au lieu de 11 , avec toutes les démarches administratives que cela comprend, aurait tout simplement dépassé les ressources temporelles et personnelles disponibles. D'autre part, il est probable que seul un nombre très restreint de dossiers nous ait échappé pour cette raison. En effet, pour participer au programme, il fallait être domicilié dans l'un des cantons participant aux essais : de plus, parmi ces cantons figurent ceux qui ont connu une importante scène ouverte de la drogue. Il est dès lors improbable qu'un nombre considérable de délits ait été commis en dehors de ce territoire.

13. Pour ces personnes, une évaluation parallèle (mais plus sommaire) a été menée dont les résultats sont similaires à ceux présentés ici (voir Killias et Rabasa, 1997). 
T A B LE A U 6

Taux d'incidence des contacts avec la police, par type de délit, durant les six mois précédant et suivant le début du traitement $(n=604)$

\begin{tabular}{|c|c|c|c|}
\hline Type de délit & Avant & Après & $p^{*}$ \\
\hline Lésions corporelles et agressions sexuelles & 0,023 & 0,022 & N.S. \\
\hline Vol à l'étalage & 0,164 & 0,078 & $<, 01$ \\
\hline Cambriolage & 0,041 & 0,013 & $<, 02$ \\
\hline Brigandage/vol à l'arraché & 0,012 & 0,002 & 06 \\
\hline Violation de domicile & 0,028 & 0,007 & $<, 02$ \\
\hline Vol de véhicules & 0,048 & 0,020 & $<, 03$ \\
\hline Autres vols et délits contre la propriétéa & 0,139 & 0,033 & $<, 01$ \\
\hline Autres délits prévus dans le code pénal ${ }^{b}$ & 0,023 & 0,007 & $<, 01$ \\
\hline Infractions à la loi sur la circulation routière & 0,040 & 0,013 & N.S. \\
\hline Consommation/possession de cannabis & 0,131 & 0,056 & $<, 01$ \\
\hline Consommation/possession d'héroïne & 0,689 & 0,149 & $<, 01$ \\
\hline Consommation/possession de cocaïne/ecstasy & 0,285 & 0,132 & $<, 01$ \\
\hline $\begin{array}{l}\text { Consommation/possession d'autres } \\
\text { ou plusieurs substances }\end{array}$ & 0,166 & 0,025 & $<, 02$ \\
\hline Trafic de drogues & 0,119 & 0,051 & $<, 01$ \\
\hline Infractions prévues dans d'autres lois ${ }^{c}$ & 0,017 & 0,005 & 07 \\
\hline Total & 1,92 & 0,61 & $<, 01$ \\
\hline
\end{tabular}

^ Test de Student (T-test) pour observations pairées.

a. Y compris faux-monnayage et recel.

b. Y compris resquille dans les transports publics.

c. Y compris demandes de recherche de la personne.

et après le début du traitement. L'âge médian de ces personnes au moment de leur entrée dans le programme était de 30 ans et la moyenne, légèrement inférieure à 31 ans.

Le tableau met en évidence une réduction très marquée du nombre de contacts avec la police à partir du début du traitement. Dans l'ensemble, la diminution est de $68 \%$ (en chiffres absolus, on passe de 1162 à 370 contacts enregistrés) et concerne pratiquement tous les délits à l'exception des lésions corporelles et des agressions sexuelles qui, elles, se maintiennent à un niveau relativement stable. Cela s'explique par le fait qu'il s'agit d'infractions éloignées du style de vie des toxicomanes, comme le confirment les données autoreportées concernant la délinquance et la 
victimisation (voir plus haut). Pour les autres catégories de délits, la diminution est statistiquement significative dans presque tous les cas : et même lorsqu'elle ne l'est pas, comme dans le cas des infractions à la loi sur la circulation routière, elle est supérieure à $50 \%{ }^{14}$.

Plus particulièrement, on soulignera ici la remarquable diminution des vols et autres délits contre la propriété qui implique une amélioration de la qualité de vie non seulement pour les toxicomanes mais aussi pour l'ensemble de la population. En outre, on constate que la police continue de poursuivre de manière très importante la simple consommation de drogues, corroborant ainsi le fait que les essais de prescription médicale d'opiacés n'ont pas conduit à un relâchement de l'action de la police sur le terrain (Killias et al., 1998). La diminution de cette catégorie de délits met aussi en évidence le fait que la prescription d'héroïne conduit à une diminution de la consommation de tout type de drogue, contrairement à ce que prévoyaient ceux qui soutenaient que les toxicomanes étaient très souvent des polytoxicomanes qui continueraient à consommer d'autres drogues en marge de l'héroïne prescrite. Ce résultat est aussi confirmé par les données autoreportées sur la consommation de stupéfiants (Uchtenhagen, 1997). Finalement, on soulignera également la réduction du trafic de drogues qui peut s'avérer particulièrement intéressante quant à la prévention, puisque les toxicomanes trafiquants jouent un rôle très important dans le recrutement de nouveaux jeunes toxicomanes.

Notons aussi que l'un des résultats les plus significatifs de cette recherche est que la diminution de la délinquance ne se limite pas au court terme. Au contraire, elle se poursuit même après 24 mois de traitement. Le Tableau 7 illustre cette affirmation en présentant la diminution des taux de prévalence et d'incidence pour différentes périodes de temps. L'échantillon se réduit au fur et à mesure que l'on avance dans le temps, puisque seule une minorité de patients avaient été traités durant au moins deux ans au moment de la récolte des données.

Le Tableau 7 révèle que la diminution de la prévalence de la délinquance — c'est-à-dire du pourcentage de personnes qui continuent à commettre des délits — se situe autour de $40 \%$ pour toutes les périodes

14. Le test de Student pour observations pairées permet de comparer de manière individuelle les scores des individus durant les six mois avant et après l'entrée en traitement. Ainsi, même lorsque les moyennes sont semblables - par exemple dans le cas des cambriolages et des infractions à la loi sur la circulation routière - les distributions ne le sont pas forcement et par conséquent la comparaison avant/après peut être significative dans un cas et ne pas l'être dans l'autre. 
T A B LEA U 7

Diminution des taux de prévalence et d'incidence de la délinquance enregistrée par la police après le début du traitement

\begin{tabular}{|ccc|}
\hline Période d'observation & Prévalence & Incidence \\
\hline 6 mois $(n=604)$ & $-43,2 \%$ & $-68,2 \%$ \\
12 mois $(n=336)$ & $-40,1 \%$ & $-68,4 \%$ \\
18 mois $(n=153)$ & $-41,2 \%$ & $-69,7 \%$ \\
24 mois $(n=108)$ & $-39,3 \%$ & $-71,1 \%$ \\
\hline
\end{tabular}

de temps étudiées. D'autre part, la diminution de l'incidence est largement supérieure à $60 \%$ : elle se situe même au delà de $70 \%$ après 24 mois de traitement, même si dans ce cas-là la taille réduite de l'échantillon rend plus difficile la généralisation des résultats. La différence entre la diminution de la prévalence et de l'incidence s'explique par le fait que les toxicomanes délinquants sont responsables d'un grand nombre de délits. Ainsi, les données de police confirment que les personnes encore impliquées dans la délinquance ont réduit de manière notoire la quantité de délits commis.

\section{Les condamnations}

Les données concernant les condamnations pénales sont conservées en Suisse dans un registre fédéral connu sous le nom de Casier judiciaire central. Toutes les condamnations pour crimes et délits, ainsi que celles pour contraventions se soldant par une peine privative de liberté ou une amende dépassant 500 francs, y sont inscrites. Pour ce faire, il existe un formulaire uniforme sur lequel les tribunaux notent les données personnelles de l'auteur, le délit pour lequel il a été condamné, la date de commission de ce délit et la peine infligée. Une copie de ce formulaire est envoyée au Casier judiciaire central où l'on constitue des dossiers personnels qui ont d'ailleurs été consultés par les collaborateurs de l'IPSC lors de la récolte des données.

Comme cette récolte a eu lieu quelques mois après celle des données relatives aux corps de police et que les casiers judiciaires de tous les participants aux essais ont été consultés, l'échantillon présenté dans nos analyses differe de celui utilisé lors de l'analyse des données de police. En effet, en ce qui concerne les patients ayant reçu de l'héroïne durant 
T A B LEA U 8

Évolution des taux de prévalence et d'incidence des condamnations pénales durant les douze mois précédant et suivant le début du traitement $(n=561)$

\begin{tabular}{|lccc|}
\hline Indicateur & Avant & Après & $p^{*}$ \\
\hline Prévalence des condamnations & 30,3 & 6,2 & $<, 01$ \\
Incidence des condamnations & 0,44 & 0,07 & $<, 01$ \\
Incidence des délits inclus dans les sentences & 0,87 & 0,12 & $<, 01$ \\
\hline
\end{tabular}

^ Test de Student (T-test) pour observations pairées.

au moins douze mois lors de la récolte des données, l'échantillon comprend 561 personnes dont les âges médian et moyen sont équivalents à ceux de l'échantillon utilisé pour les données de police.

Le Tableau 8 permet d'avoir un aperçu de l'évolution des inscriptions au casier judiciaire pour la période de référence de douze mois avant et après le début du traitement. Il présente en détail l'évolution du nombre de personnes condamnées, du nombre moyen de condamnations, ainsi que du nombre moyen de délits inclus dans les condamnations. Ce dernier indicateur est aussi important du fait que, comme le montre le tableau, plusieurs délits sont souvent inclus dans une même sentence.

Les données montrent une nette diminution autant du nombre de condamnations inscrites que du nombre de délits respectifs. De plus, la grande majorité des participants est restée sans nouvelle inscription durant les 12 mois suivant le début du traitement. À ce sujet, il est important de signaler que lors du codage des extraits de casier judiciaire, les délits ont été attribués à la période avant ou après l'entrée dans le programme selon la date de commission du délit et non selon la date de la sentence qui se situe forcément un peu plus tard. Ainsi, le risque existe que des délits commis durant la période de référence n'aient pas encore fait l'objet d'une condamnation au moment de la récolte des données. Pour contrôler ce facteur, nous avons aussi étudié l'évolution de la délinquance durant les 12 mois précédant et suivant le début du traitement des personnes traitées au moins 24 mois au moment de la récolte des données $(n=136)$. De cette manière, les tribunaux disposent d'une période additionnelle d'une année pour juger le délit. Cette analyse n'a toutefois pas montré de différences significatives avec les résultats présentés dans le tableau 8 . Dans les deux cas, la diminution de la délinquance se situe autour de $80 \%$ pour tous les indicateurs retenus. Cela suggère une diminution de la gravité des délits commis, puisqu'il semble 
T A B LEA U 9

Évolution de la gravité des condamnations pénales durant les douze mois précédant et suivant le début du traitement $(n=561)$

\begin{tabular}{|lcc|}
\hline Indicateur & Avant & Après \\
\hline Incidence des condamnations à des peines de prison & 0,39 & 0,06 \\
Durée moyenne des peines de prison imposées (en jours) & 76,1 & 34,1 \\
Moyenne des jours de prison imposés & 29,8 & 2,1 \\
Moyenne des jours passés en détention préventive & 3,0 & 0,1 \\
\hline
\end{tabular}

évident que certaines infractions découvertes par la police n'ont pas donné lieu à des condamnations inscrites dans le casier judiciaire central.

Afin d'analyser de manière plus approfondie cette hypothèse, nous avons calculé le nombre de condamnations à des peines de prison parmi les condamnations imposées, ainsi que la durée moyenne de ces peines. En outre, nous avons relevé le nombre de jours de prison imposés et de jours passés en prison préventive. L'évolution de ces indicateurs pour des périodes de 12 mois précédant et suivant le début du traitement est présentée dans le Tableau 9.

Ce tableau confirme que la prescription d'héroïne conduit non seulement à une diminution de la délinquance, mais aussi à une diminution de la gravité des délits commis, puisque la durée moyenne des peines de prison imposées s'est réduite de manière significative après le début du traitement. En outre, la diminution du nombre de jours de prison imposés ainsi que de jours passés en détention préventive est très impressionnante et devrait avoir sans doute une influence sur les coûts liés à l'administration de la justice. Signalons finalement que nous avons renoncé à une analyse répartie selon les différents délits du fait du nombre restreint de condamnations pour la période suivant le début du traitement.

\section{Conclusions}

L'analyse des résultats de cette recherche indique que la prescription médicale d'héroïne conduit à une diminution massive de la délinquance des toxicomanes traités. Avant leur entrée dans le programme, ces derniers constituaient une population extrêmement ancrée dans la délinquance : mais le traitement a permis de réduire notamment le pour- 
centage de personnes délinquantes et - de manière encore plus importante - le nombre de délits commis.

À ce sujet, la convergence sans équivoque des différents indicateurs de la criminalité utilisés mérite d'être signalée. En effet, il est difficile d'imaginer un problème de validité qui aurait pu affecter ces indicateurs dans leur ensemble. D'ailleurs, les premiers résultats d'une comparaison plus détaillée entre les données officielles et les données de sondages semblent confirmer leur validité (Aebi, 1999).

Plus particulièrement, les sondages de délinquance autoreportée enregistrent une diminution d'environ $90 \%$ de la fréquence des délits contre la propriété et de la vente de drogues dures. En revanche, la diminution est faible pour les délits peu liés au style de vie des héroïnomanes, à savoir les délits impliquant une certaine violence.

En ce qui concerne les données du casier judiciaire, ces dernières permettent de constater une diminution d'environ $80 \%$ des condamnations qui y sont inscrites. À ce niveau, on constate aussi des diminutions très importantes pour ce qui est des jours de prison imposés ainsi que des jours passés en prison préventive. En outre, la durée moyenne des peines de prison imposées s'est réduite de plus de $50 \%$.

En ce qui concerne les dossiers de police, le pourcentage de toxicomanes engagés dans la délinquance s'est réduit de $40 \%$, tandis que la diminution du nombre de délits enregistrés dépasse largement $60 \%$. Cette diminution ne se limite pas au court terme : au contraire, elle se maintient même après 24 mois de traitement.

Finalement, les sondages de victimisation renforcent la validité de ces résultats. En effet, on y enregistre une forte diminution des expériences de victimisation, notamment en ce qui concerne les délits liés au style de vie des toxicomanes. Cette diminution est plus marquée pour les personnes les plus ancrées dans la délinquance, confirmant ainsi le lien entre délinquance et victimisation.

Dans l'ensemble, on remarquera également la très importante diminution des vols et des délits contre la propriété ainsi que du trafic de stupéfiants et de la consommation d'autres drogues illégales en marge de l'héroïne prescrite. Ces résultats mettent en évidence qu'en dehors de l'amélioration de l'état de santé des toxicomanes (voir Uchtenhagen, 1997), la prescription d'héroïne peut aussi s'avérer une mesure très efficace quant à de la prévention de la délinquance et entraîner une amélioration de la qualité de vie de l'ensemble de la population à travers la diminution des délits commis. En effet, comparativement aux résultats 
d'autres programmes de prévention de la criminalité, les réductions observées dans le cadre des essais avec prescription médicale de stupéfiants méritent d'être qualifiées d'exceptionnelles.

Il semble aussi important de souligner la diminution de la vente de drogues dures, qui est selon les dires des participants au programme l'un des délits les plus souvent commis. En effet, il semble raisonnable de présumer que c'est par ce biais que le recrutement d'une grande partie des nouveaux consommateurs est assuré. On peut donc espérer que les essais de prescription médicale de stupéfiants contribueront également à réduire la perpétuation de la toxicodépendance, au-delà de la réduction des dommages collatéraux.

Notons pour conclure que la qualification d'un programme en termes de réussite ou d'échec dépend, entre autres, des critères d'évaluation retenus. Si l'abandon total de la consommation de stupéfiants n'est pas pris comme unique objectif, autrement dit si l'amélioration de la situation sociale et médicale des personnes traitées ainsi que la réduction des dommages causés à la société tout entière sont également pris en considération, la réussite du programme suisse de prescription médicale d'héroïne ne semble guère contestable.

\section{Références}

AEBI, M. F. 1999. La validité des indicateurs de la criminalité: Les sondages de délinquance autoreportée face aux données de police et du casier judiciaire dans le cadre de l'évolution des essais suisses de prescription d'héroine, Thèse de doctorat en criminologie, Université de Lausanne.

Ball, J. C., Rosen, L., Flueck, J. A. et Nurco, D. N. 1981. « The criminality of heroin addicts : When addicted and when off opiates », p. 39-65 in The Drugs-Crime Connection, sous la direction de J. A. Inciardi. Beverly Hills/ London : Sage.

BARON, S. W. 1997. "Risky Lifestyles and the Link Between Offending and Victimization », Studies on Crime and Crime Prevention 6 (1) : 53-71.

Boggio, Y., Cattacin, S., Cesoni, M. L. et LuCAS, B. 1997. Apprendre à gérer: La politique suisse en matière de drogue, Genève : Georg.

Borst-EILERS, B., SORgDRAger, W. et KoHnStamm, J. 1995. Drugs Policy in the Netherlands: Continuity and Change, Rijswijk: The Central Information, Documentation and Library Archives, Directorate of the Ministry of Health, Welfare and Sport of the Netherlands.

GotTfRedson, M. R. 1984. Victims of Crime: The Dimensions of Risk, London : HMSO. 
GrapendaAl, M., LeUW, E. et Nelen, H. 1995. A World of Opportunities : LifeStyle and Economic Behavior of Heroin Addicts in Amsterdam, Albany N.Y. : Sunny Press.

Grob, P. J. 1993. « The Needle Park in Zurich : The Story and the Lessons to be Learned », European Journal on Criminal Policy and Research 1 (2) : 48-60.

Junger-Tas, J., Terlouw, G.-J. et KleIN, M. W. 1994. Delinquent Behavior Among Young People in the Western World: First Results of the International Self-Report Delinquency Study, Amsterdam/New York : Kugler.

Killias, M. 1991. Précis de criminologie, Berne : Staempfli.

Killias, M. et RaBASA, J. 1997. Rapport final sur les effets de la prescription de stupéfiants sur la délinquance des toxicomanes, Lausanne: Institut de police scientifique et de criminologie, Université de Lausanne.

Killias, M. et Ribeaud, D. 1999. « Drug Use and Crime Among Juveniles : An International Perspective », Studies on Crime and Crime Prevention 8 (2) : 189209.

Killias, M. et UChtenhagen, A. 1995. «Méthodologie de l'évaluation des essais suisses avec prescription médicale d'opiacés sous l'angle de la délinquance : l'accès sous contrôle médical à l'héroïne réduit-il la déliquance des toxicomanes? », Bulletin de criminologie 21/2 : 33-48.

Killias, M., Rabasa, J. et Villettaz, P. 1994a. "Drogenkonsum und abweichendes Verhalten », p. 183-194, Illegale Drogen und Kriminalität in der Schweiz, sous la direction de M. Eisner. Lausanne : ISPA-Press : 183-194.

Killias, M., Villettaz, P. et Rabasa, J. 1994b. «Self-Reported Juvenile Delinquency in Switzerland », p. 186-211, Delinquent Behavior Among Young People in the Western World: First Results of the International Self-Report Delinquency Study, sous la direction de J. Junger-Tas, G.-J. Terlouw et M. W. Klein. Amsterdam/New York : Kugler.

Killias, M., Aebi, M. F. et Ribeaud, D. 1998. « Effects of Heroin Prescription on Police Contacts among Drug-Addicts », European Journal on Criminal Policy and Research 6 (3) : 433-438.

Killias, M., Ribeaud, D. et AEBI, M. F. À paraître. « Drogenabhängige als Opfer von Straftaten : Was bewirkt die Verschreibung von Heroin », in Handbuch der Drogenmedizin, sous la direction de A. Uchtenhazer. Munich : Urban \& Schwarzenberg.

KreuZer, A., RÖMER-KLEES, R. et SCHNEIDER, H. 1991. Beschaffungskriminalität Drogenabbängiger, Wiesbaden : BKA.

Mayhew, P. et ElliotT, D. 1990. «Self-Reported Offending, Victimization, and British Crime Survey », Violence and Victims 5 (2) : 83-96.

OFSP — Office fédéral de la santé publique. 1997. Politique de la Confédération Suisse en matière de drogue: Des chiffres et des faits, Berne : Office fédéral de la santé publique. 
Reuband, K.-H. 1998. «Drug Policies and Drug Prevalence: The Role of Demand and Supply », European Journal on Criminal Policy and Research 6 (3) : 321-336.

RiHS-Middel, M. 1994. « Medical Prescription of Narcotics in Switzerland:

Basic Issues and Research Plan », European Journal on Criminal Policy and Research 2 (4) : 69-83.

UCHTENHAGEN, A. 1997. Essais de prescription médicale de stupéfiants : Rapport de synthèse, Zürich : Institut für Suchtforschung, Universität Zürich.

VILLMOW, B. et STEPHAN, E. 1983. Jugendkriminalität in einer Gemeinde: Eine Analyse erfragter Delinquenz und Viktimisierung sowie amtlicher Registrierung, Freiburg I. B. 